http://jmscr.igmpublication.org/home/ ISSN (e)-2347-176x ISSN (p) 2455-0450

crossref DOI: https://dx.doi.org/10.18535/jmscr/v7i9.56

\title{
Assessment of Platelet Indices as Prognostic Markers in Seropositive Cases of Dengue
}

\author{
Authors \\ Dr Indumathi $S^{1}$, Dr Anoosha $K^{2}$, Dr Lakshmi $V^{3}$ \\ ${ }^{1,2}$ Assistant Professor, Department of Pathology, Sambhram Institute of Medical Sciences and Research \\ ${ }^{3}$ Professor and Head of the Department, Sambhram Institute of Medical Sciences and Research
}

\begin{abstract}
Background: Dengue fever is the most prevalent arboviral disease globally and an increasing number of cases are being reported every year. The assessment of platelet count and platelet indices at an early stage in the disease helps to predict the life-threatening complications such as Dengue Haemorrhagic Fever and Dengue Shock Syndrome.

Materials and Methods: The current case-control study was undertaken at Sambhram Institute of Medical Sciences and Research, a rural tertiary care and teaching hospital in Kolar Gold Fields. During the recent dengue outbreak between June 2019 to August 2019 where in 75 seropositive cases of dengue were reported following clinical examination. Platelet count and platelet indices (Mean Platelet Volume, Platelet Distribution Width, Plateletcrit and Platelet-Large Cell Ratio) were carried out for these cases and results were tabulated. Similar tests were carried out on 75 apparently healthy controls and required parameters were obtained.

Results: Platelet counts ranged from $11-429 \times 10^{3} \mathrm{~mm}^{3} .58$ cases (77.33\%) had thrombocytopenia. MPV ranged from 8-14 fL. 15 cases (20\%) had increased MPV >12 fL. PDW ranged from 9-22.4. 21 cases (28\%) had increased PDW > 14fL. PCT ranged from 0.04- $0.36 \%$. 60 cases (80\%) had low plateletcrit $<0.22 \%$. P-LCR ranged from $12.8-52.4 \%$. 13 cases $(17.33 \%)$ had increased $P-L C R>35 \%$. Statistically significant $p$ values were found for Platelet count $(p<0.0001), M P V(p=0.0006)$ and PCT $(p=0.0003)$. The $p$-values for PDW (0.2966) and P-LCR (0.1229) were not found to be statistically significant.

Conclusion: Although dengue fever is predominant in urban and semi urban areas, an increasing in number of cases are being reported in rural areas. Platelet indices can be an early predictor of complications in dengue fever and thus benefit both Pathologists and Clinicians alike to forsee high-risk cases. It is an easily available, fast and cost effective means to ensure prompt management in a rural centers with limited resources. Platelet count, Mean platelet volume and Plateletcrit were found to be significant prognostic markers in the present study.

Keywords: Dengue fever, Platelet Count, Platelet Indices, Seropositive.
\end{abstract}

\section{Introduction}

Dengue fever is a global epidemic disease with half of the world's population being at- risk and an increasing number of cases being reported in
India. It is caused by an Arbovirus of family Flaviviridiae which is spread by the vector mosquito Aedesaegypti, which is a day- time feeder. The 4 distinct serotypes of the virus which 
cause dengue are DEN-1, DEN-2, DEN- 3 and DEN- 4. The period of incubation is 4-10 days. Infected individuals are the carriers and multipliers of the virus. Dengue is characterized by high fever, headache, retro-orbital pain, myalgia, arthralgia, nausea and vomiting. Although the fever is self- limiting it can lead to potentially lethal complications like Dengue Haemorrhagic Fever (DHF) and Dengue Shock Syndrome (DSS) which can present with warning signs such as rash, petechial haemorrhage, mucosal bleeding, melena, respiratory distress, fluid accumulation and Disseminated Intravascular Coagulation (DIC). The following 24-48 hours require proper monitoring and medical care to avoid the risk of death. Thus, the fever is manifest in 3 stages- Febrile, critical and recovery. ${ }^{[1,2,3,4]}$

Thrombocytopenia is an important feature of acute infection. It results due to increased destruction, reduced production and splenic sequestration. Monitoring of platelet counts at regular intervals becomes mandatory as it is an important aid in the prediction of complications. Severe thrombocytopenia is directly correlated to disease severity, chances of development of complications and prognosis in the critically-ill patients. ${ }^{[2,5]}$

Platelet indices include Mean Platelet Volume (MPV), Platelet Distribution Width (PDW), Plateletcrit (PCT) and Platelet- Large Cell Ratio (P-LCR). MPV measures the average size of platelets and provides a statement between the lower discriminator (PL) and upper discriminator (PU). PDW is a measure of anisocytosis of platelets. Plateletcrit is the sum of platelet impulses which are individually detected by impedance measurement. P-LCR indicates the percentage of large platelets with volume $>12 \mathrm{fL}$. An increase is an indication for platelet aggregates, giant platelets and regenerating large platelets. ${ }^{[6,7]}$

The present study aims to understand the utility of the various platelet parameters in the prognosis of dengue fever in a rural area where the number of seropositive cases of dengue are exponentially on the rise.

\section{Materials and Methods}

The present case- control study was undertaken at the Central Laboratory of Sambhram Institute of Medical Sciences and Research which is a rural tertiary care and teaching hospital in Kolar Gold Fields during the recent dengue outbreak between June 2019 to August 2019 during which 75 seropositive cases of dengue were diagnosed based on clinical, serological and hematological parameters. Blood samples of all the cases were collected in K3 EDTA tubes and the platelet indices were obtained using semi- automated Sysmex XP-100 hematology analyzer. The samples were processed within 1 hour of collection. Cases with platelet count less than 1.50 lakhs $/ \mathrm{mm}^{3}$ were confirmed with peripheral smear examination. Control population comprised of 75 apparently healthy individuals with no underlying symptoms of dengue or pre-existing diseases capable of producing thrombocytopenia. Similar tests were carried out on the control group The serological tests for dengue NS1 antigen, IgG and IgM antibodies were carried out using Rapid Visual card test (J. Mitra and Co. Pvt. Ltd) and results were tabulated using a Microsoft excel sheet.

Statistical analysis- The tabulated data was analysed using unpaired T-test and $\mathrm{p}$ value was calculated using mean and standard deviation of various parameters for cases and controls. $\mathrm{P}$ value $<0.05$ was considered to be statistically significant Inclusion Criteria- All seropositive cases of dengue (NS1, IgG and IgM) in all age groups Exclusion Criteria- Thrombocytopenia resulting from causes other than dengue (seronegative)

\section{Results}

During the study period of 3 months, 75 cases were identified to be seropositive for dengue, age ranging between 2 to 68 years among which $50(66.67 \%)$ were males and 25(33.33\%) were females. Male to female ratio was 2: 1.75 
apparently healthy individuals with normal platelet counts and indices were chosen as controls with age ranging between 14 to 50 years among which 60(80\%) were males and 15(20\%) were females. Male to female ratio was $4: 1$.

33 cases (44\%) were found to be in the age group of $20-39$ years followed by 16 cases $(21.33 \%)$ in the age group of 40-59 years. In the control group, 52 individuals $(69.33 \%)$ were in the age group between 20-39 years. (Tables 1, 2 and 3)

Table 1- Age range in cases and controls

\begin{tabular}{|l|c|c|}
\hline Parameters & $\begin{array}{c}\text { Cases } \\
\text { (in years) }\end{array}$ & $\begin{array}{c}\text { Controls } \\
\text { (in years) }\end{array}$ \\
\hline Age range & $2-68$ & $14-50$ \\
\hline
\end{tabular}

Table 2- Prevalence of cases and controls by sex

\begin{tabular}{|l|c|c|}
\hline Sex & Cases (n /\%) & Controls (n /\%) \\
\hline Males & $50(66.67 \%)$ & $60(80 \%)$ \\
\hline Females & $25(33.33 \%)$ & $15(20 \%)$ \\
\hline Male:Female Ratio & $2: 1$ & $4: 1$ \\
\hline
\end{tabular}

Table 3- Prevalence of cases and controls by age groups

\begin{tabular}{|l|c|c|}
\hline $\begin{array}{l}\text { AGE GROUPS } \\
\text { (in years) }\end{array}$ & $\begin{array}{c}\text { CASES } \\
(\mathbf{n} / \%)\end{array}$ & CONTROLS (n /\% ) \\
\hline $0-19$ & $15(20 \%)$ & $11(14.67 \%)$ \\
\hline $20-39$ & $33(44 \%)$ & $52(69.33 \%)$ \\
\hline $40-59$ & $16(21.33 \%)$ & $12(16 \%)$ \\
\hline $60-79$ & $11(14.67 \%)$ & $00(0 \%)$ \\
\hline
\end{tabular}

Among the 75 seropositive cases, 50 (66.67\%) were positive for NS1 antigen, $28(37.33 \%)$ were positive for IgG antibody and $47(62.67 \%)$ were positive for Ig M antibody. Seropositivity for both antigen and antibody (NS1, IgG and $\operatorname{IgM}$ ) was observed in 14 cases (18.67\%). (Table 4)

Table 4- Seropositivity for dengue

\begin{tabular}{|l|c|c|}
\hline $\begin{array}{l}\text { ANTIGEN/ } \\
\text { ANTIBODY }\end{array}$ & $\begin{array}{c}\text { SEROPOSITIVE } \\
(\mathbf{n} / \%)\end{array}$ & $\begin{array}{c}\text { SERONEGATIVE } \\
(\mathbf{n} / \%)\end{array}$ \\
\hline NS 1 antigen & $50(66.67 \%)$ & $25(33.33 \%)$ \\
\hline IgG antibody & $28(37.33 \%)$ & $47(62.67 \%)$ \\
\hline IgM antibody & $47(62.67 \%)$ & $28(37.33 \%)$ \\
\hline
\end{tabular}

Among these cases, platelet counts ranged from $11-429 \times 10^{3} \mathrm{~mm}^{3}$ (Normal range- 150-450 x $10^{3}$ $\left.\mathrm{mm}^{3}\right) .58$ cases $(77.33 \%)$ had thrombocytopenia $\left(<150 \times 10^{3} \mathrm{~mm}^{3}\right)$. MPV ranged from 8-14 fL. (Normal range- 8-12 fL). 15 cases (20\%) had increased MPV >12 fL. PDW ranged from 9-22.4 (Normal range 9-14fL). 21 cases (28\%) had increased PDW > 14fL. PCT ranged from 0.04$0.36 \%(0.22-0.24 \%)$. 60 cases $(80 \%)$ had low plateletcrit $<0.22 \%$. P-LCR ranged from 12.8-52.4 $\%$ (Normal range-15-35\%). 13 cases (17.33\%) had increased P-LCR $>35 \%$. (Table 5).

$\mathrm{P}$ - values obtained using unpaired $\mathrm{T}$ - test yielded statistically significant values for Platelet count (<0.0001), MPV (0.0006) and PCT (0.0003). However, the p- values for PDW (0.2966) and PLCR (0.1229) were not found to be statistically significant. (Table 5)

Table 5- Statistical evaluation of platelet indices in cases and controls

\begin{tabular}{|c|c|c|c|}
\hline $\begin{array}{l}\text { Platelet } \\
\text { Indices }\end{array}$ & Cases & Controls & P Value \\
\hline \multicolumn{4}{|c|}{ PLATELET COUNT $\left(\times \mathbf{1 0}^{3} \mathrm{~mm}^{3}\right)$} \\
\hline Range & $11-429$ & $148-441$ & \multirow[t]{4}{*}{$<0.0001$} \\
\hline Mean & 133 & 299.57 & \\
\hline Median & 118 & 301 & \\
\hline $\mathrm{SD}$ & 295 & 207.18 & \\
\hline \multicolumn{4}{|c|}{ MPV (in fL) } \\
\hline Range & $8-14$ & $7.9-12.7$ & \multirow[t]{4}{*}{0.0006} \\
\hline Mean & 10.4 & 8.2 & \\
\hline Median & 10.3 & 8.0 & \\
\hline SD & 4.24 & 3.39 & \\
\hline \multicolumn{4}{|c|}{ PDW ( in fL) } \\
\hline Range & $9-22.4$ & $8.9-19.3$ & \multirow[t]{4}{*}{0.2966} \\
\hline Mean & 12.97 & 11.52 & \\
\hline Median & 12.40 & 10.9 & \\
\hline SD & 9.47 & 7.35 & \\
\hline \multicolumn{4}{|c|}{ Plateletcrit (in \%) } \\
\hline Range & $0.04-0.36$ & $0.13-0.44$ & \multirow[t]{4}{*}{0.0003} \\
\hline Mean & 0.13 & 0.28 & \\
\hline Median & 0.13 & 0.27 & \\
\hline SD & 0.22 & 0.21 & \\
\hline \multicolumn{4}{|c|}{ P-LCR (in \%) } \\
\hline Range & $12.8-52.4$ & $11.2-38.6$ & \multirow[t]{4}{*}{0.1229} \\
\hline Mean & 27.68 & 21.58 & \\
\hline Median & 26.80 & 20.07 & \\
\hline SD & 28.00 & 19.37 & \\
\hline
\end{tabular}

2 patients ended up with Dengue Hemorrhagic Fever and were found to have a positive tourniquet test. Complications such a petechial rashes, mucosal bleeding, vomiting, hypotension and malena resulted. These 2 patients were found to be seropositive for NS1, IgG and IgM. They were also found to have severe thrombocytopenia of 11 and 20x $10^{3} \mathrm{~mm}^{3}$ respectively. Peripheral smear showed severe thrombocytopenia and giant platelets (Figure 1\& 2). MPV was 13.5 and 14fL. PDW was 20.3 and 22.4fL. PCT was 0.04 and 
$0.08 \%$ and P-LCR was 49.3 and $52.4 \%$ respectively. Both the patients were referred to higher centers and thus lost to follow-up.

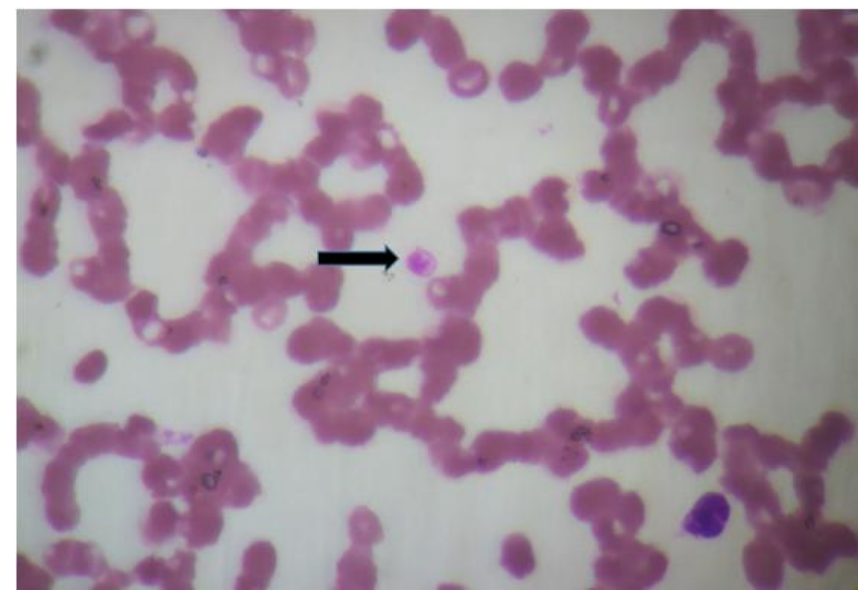

Figure 1- Peripheral smear showing severe thrombocytopenia (platelet count-11 x $10^{3} \mathrm{~mm}^{3}$ ) with giant platelet (black arrow), (Leishman stain, $\mathrm{x}$ 1000)

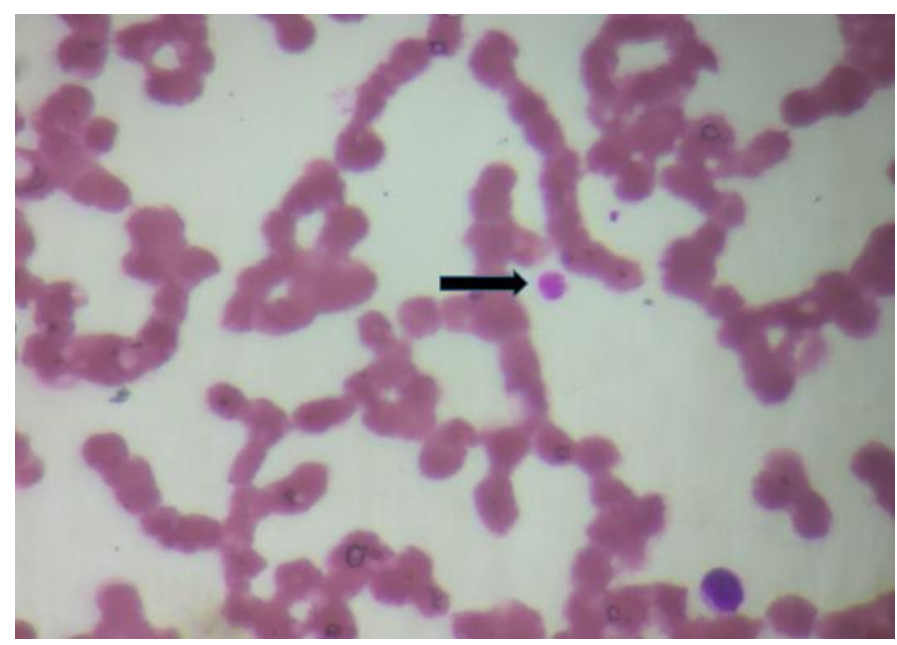

Figure 2- Peripheral smear showing severe thrombocytopenia (platelet count-20 x 10 $\mathrm{mm}^{3}$ ) with giant platelet (black arrow), (Leishman stain, $\mathrm{x}$ 1000)

\section{Discussion}

The incidence of dengue fever was predominant in males in the present study with $66.66 \%$ cases which was similar to the study done by Agarwal R et al and Anker $\mathrm{M}$ et al who also found a male predominance. $^{[8,9]}$

Maximum number of cases (44\%) were observed in the 20-39 years age group in the present study which was similar to the study conducted by Cecilia D et al who also reported in their 6 year study that the most commonly affected age group was 21-40 years. Afsar $\mathrm{N}$ et al also reported $86.7 \%$ of cases in their study to be under 30 years of age. ${ }^{[5,10]}$

Thrombocytopenia indicates a fall in platelet count to $<150 \times 10^{3} \mathrm{~mm}^{3}$. Slichter $\mathrm{SJ}$ et al suggested that dengue virus induces anti-platelet antibodies that cause vigorous platelet destruction through apoptosis and lysis by complement system. The virus has a Non- Structural protein 1 (NS1) against which the antibodies produced in dengue fever act damaging the platelets and endothelial cells. Study by Anuradha $\mathrm{M}$ et al showed $89 \%$ cases with thrombocytopenia which was slightly more than the present study $(77.33 \%){ }^{[11,12,13]}$

In the study conducted by Manoharan A et al, MPV was found to be high in patients with bleeding manifestations which similar to the present study. Afsar $\mathrm{N}$ et al found a statistically significant $p$ value in their study of MPV which was concurrent with our study. Increased platelet volume increases platelet adhesion, aggregation and synthesis of thromboxanes which can lead to complications like disseminated intravascular coagulation. $^{[2,5]}$

Prakash GM et al found no correlation between increased PDW and dengue fever which was concurrent with the present study where a statistical significant value was not obtained. Nehara HR et al suggested that high PDW is more sensitive for dengue hemorrhagic fever and more specific for dengue fever. ${ }^{[14,15\}}$

A statistically significant $p$ value of 0.0003 was obtained for plateletcrit in the present study which was similar to the observation made by Nehara $\mathrm{HR}$ et al who also found a significant positive correlation. (p value $<0.0001) .{ }^{[15]}$

Bashir AB et al found a positive correlation for $\mathrm{P}$ LCR in their case-control study ( $p$ value- 0.000 ) where P-LCR was increased in cases with thrombocytopenia which was not in concurrence with the present study. ${ }^{[16]}$ 


\section{Conclusion}

Owing to the increasing global burden of dengue epidemic, novel platelet indices such as MPV, PDW, PCT and P-LCR can be used as markers of platelet activity thus playing a pivotal role to predict the complications such as DHF and DSS. The present study of 75 seropositive cases for dengue found a highly significant correlation between platelet count, MPV and PCT as prognostic markers.

\section{References}

1. WHO. Dengue hemorrhagic fever: Diagnosis, treatment, prevention and control. Geneva: WHO press; 2009:3-106.

2. Manoharan A, Sinha P. Role of mean platelet volume in dengue fever aprognostic marker. Intl $\mathrm{J}$ Res Rev 2017;4(6):72-77.

3. Sharma K, Yadav A. Association of mean platelet volume with severity, serology and treatment outcome in dengue fever: Prognostic utility. J Clin Diagn Res2015;9 (11):1-3.

4. Dengue: Guidelines for diagnosis, treatment, prevention, and control in subSaharan Africa and 13 countries in SouthAmerica. Geneva: World Health Organization; 2009. WHO.

5. Afsar N, Afroze IA, Humaira S, Abid Z. Use of mean platelet volume as an initial diagnostic marker in evaluation of dengue fever. Ann Path Lab Med 2017;4(3):31013.

6. Wintrobe's Clinical Hematology, 11th edition: 632.

7. Dacie and Lewis Practical Hematology, $11^{\text {th }}$ edition:47-48.

8. Agarwal R, Kapoor S, Nagar R, Misra A, Tandon R,Mathur A, et al. A clinical study of the patients withdengue hemorrhagic fever during the epidemic of 1996 at Lucknow, India. Southeast Asian J Trop Med Public Health1999;30:735-40.
9. Anker M, Arima Y. Male-female differences in the number ofreported incident dengue fever cases in six Asian countries.Western Pacific Surveillance and Response Journal 2011;2(2):17-23.

10. Cecilia D. Current status of dengue and chikungunya in India. WHO South-East Asia Journal of Public Health 2014;3(1):22-27.

11. Slichter SJ, Davis K, Enright H, Braine H, Gernsheimer T, Kao K-J, et al. Factors affecting post transfusion platelet increments, platelet refractoriness, and platelet transfusion intervals in thrombocytopenic patients. Blood 2005;105(10):4106-14.

12. Lin CF, Wan SW, Cheng HJ, Lei HY,L in YS. Autoimmune pathogenesis indengue virus infection. Viral Immunol 2006;19:127-132.

13. Anuradha M, Dandekar RH. Screening and manifestations of seropositive dengue fever patients in Perambalur: a hospital based study. Intl J Med Sci and Public Health 2014;3(6):745-48.

14. Prakash GM, Anikethana G V. Use of mean platelet volume and platelet distributionwidth in predicting trend in platelet count and bleedingrisks in patients of dengue fever. Int $\mathrm{J}$ Adv Med 2016;3:611-3.

15. Nehara HR, Meena SL, Parmar S, Gupta BK. Evaluation of platelet indices in patients with dengue infections. Intl $\mathrm{J}$ Scientific Res 2016;5(7):78-81.

16. Bashir AB, Saeed OK, Mohammed BA, Ageep AK. Role of platelet indices in patients with dengue infection in Red Sea State, Sudan. Intl J Sci Res 2015;4:1573-6. 